\title{
Keyword Based Emotion Word Ontology Approach for Detecting Emotion Class from Text
}

\author{
Ashish V C ${ }^{1}$, Somashekar $\mathbf{R}^{2}$, Dr. Sundeep Kumar $\mathbf{K}^{3}$ \\ ${ }^{1}$ Computer Science and Technology, M.Tech, SEA College of Engineering \& Technology, Bangalore, India \\ ${ }^{2}$ M. Tech, Assistant Professor, SEA College of Engineering\& Technology, Bangalore, India \\ ${ }^{3}$ M. Tech (IT),M.E.(CSE), Ph.D(CSE), Professor \& HOD (PG), Department of Computer Science \& Engineering, \\ SEA College of Engineering \& Technology, Bangalore, India
}

\begin{abstract}
Human Computer interaction is a very powerful and most current area of research because the human world is getting more digitize. This needs the digital systems to imitate the human behavior correctly. Emotion is one aspect of human behavior which plays an important role in human computer interaction, the computer interfaces need to recognize the emotion of the users in order to exhibit a truly intelligent behavior. Human express the emotion in the form facial expression, speech, and writing text. Every day, massive amount of textual data is gathered into internet such as blogs, social media etc. This comprises a challenging style as it is formed with both plaint text and short messaging language. This paper is mainly focused on an overview of emotion detection from text and describes the emotion detection methods. These methods are divided into the following four main categories: keyword-based, Lexical Affinity method, learning based, and hybrid based approach. Limitations of these emotion recognition methods are presented in this paper and also, address the text normalization using different handling techniques for both plaint text and short messaging language.
\end{abstract}

Keywords: Digital systems, Human Behavior, Emotion, Intelligent Behavior, Human Express, plain text and Hybrid Based Approach

\section{Introduction}

Emotion is energy-in-motion. It is a way of expressing oneself in life. Emotions are essential part of human interaction. The emotion and sentiment recognition mechanism has been implemented in many kinds of media some of them are facial expressions, speech, image, and textual data and so on. Among these textual data is of great importance to the researchers. Due to its small storage medium textual data is the most appropriate medium for network transmission. Now a day's most of the communication is performed through text. This kind of communication includes SMS communication, chat communication, emails as well as review or feedback based communication. .A wide range of research work is related to emotions and sentiments in fields like communication, psychology, linguistics. Basically the emotions are divided into two broad types i.e. Positive and Negative. There are six basic categories of emotions namely happiness, sadness, anger, disgust, surprise and fear. Examples of positive emotions include happiness, laughter, and interest and so on. Negative emotions include anger, fear, sadness etc.

Emotions are an important aspect in the interaction and communication between people. The exchange of emotions through text messages and posts of personal blogs poses the informal style of writing challenge for researches. Extraction of emotions from text can applied for deciding the human computer interaction which governs communication and many more [1]. Emotions may be expressed by a person's speech, facial and text based emotion respectively.

Emotions may be expressed by one word or a bunch of words. Sentence level emotion detection method plays a crucial role to trace emotions or to search out the cues for generating such emotions. Sentences are the essential information units of any document. For that reason, the document level emotion detection method depends on the emotion expressed by the individual sentences of that document that successively relies on the emotions expressed by the individual words. Emotions could be expressed by a person's speech, face expression and text. Globally, the emotions are divided into six types which are joy, love, surprise, anger, sadness and fear [2]. Sufficient amount of work has been done related to speech and facial emotion detection but text based emotion recognition system still requires attraction of researchers. The short messaging language has the ability to interrupt and falsify Natural language processing tasks done on text data. To illustrate that ability, Consider an example, "At de moment he can't just put me in ad better zone though. Happy bday mic, ur a legend".

\section{Text Based Emotion Recognition Methods}

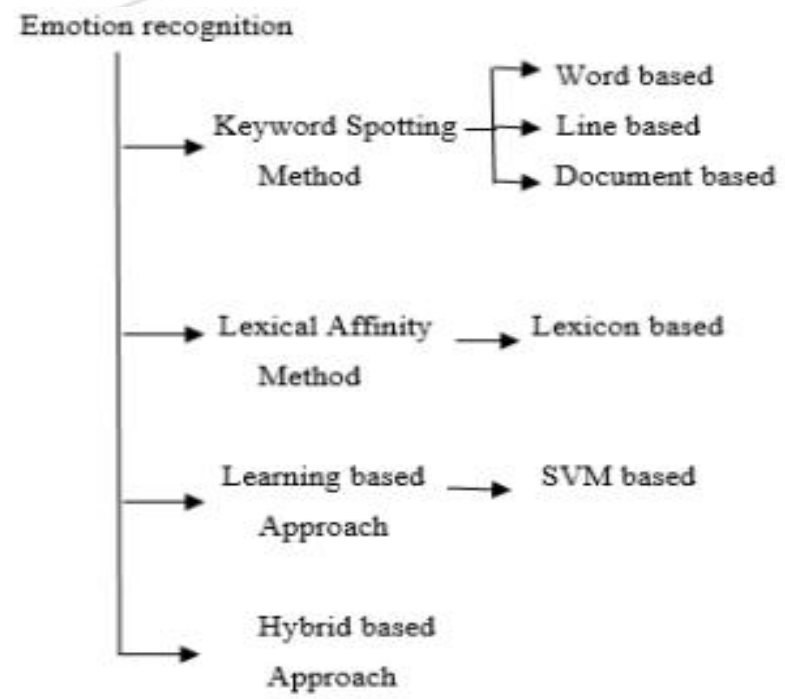

Figure 1: Text based emotion recognition techniques 


\section{International Journal of Science and Research (IJSR) \\ ISSN (Online): 2319-7064 \\ Index Copernicus Value (2013): 6.14 | Impact Factor (2015): 6.391}

There are four different text based emotion recognition techniques: Keyword spotting method, Lexical Affinity Method, learning based method and hybrid methods [5]. These methods are divided into sub-categories as shown in above figure.

\subsection{Keyword Spotting Method}

This approach is easy to implement and intuitive since it involves identifying words to search for in text [6]. It. The keyword pattern matching problem can be described as the problem of finding occurrences of keywords from a given set as substrings in a given string. These words are classified into categories such as disgust, sadness, happy, anger, fear, surprise etc. Keyword spotting method is shown as below in the figure

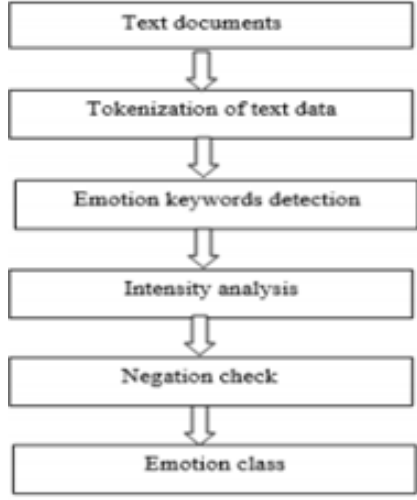

Figure 2: Keyword spotting method
The keyword spotting technique use different methods like Word based Keyword Spotting, Line based Keyword Spotting and Document based Keyword Spotting [7].

\subsection{Lexical Affinity Method}

Lexical Affinity methods are an extension of keyword spotting method. This assigns a probabilistic affinity for a particular emotion to arbitrary words rather than detecting predefined emotional keywords from text. The probabilities which are assigned by this method are part of linguistic corpora. IT has some disadvantages are the assigned probabilities are biased toward corpus specific genre of texts and does not recognize the emotions from the text that not resides at the word-level on which this method operates.

Consider an example,

"I met my old classmate by accident".

In the above sentence the word "accident" is indicates the high probability which having a negative emotion. But exact situation in this sentence that accident word not showing negative emotional assessment.

\subsubsection{Lexicon-Based Approach [8]}

Lexicon-based approaches based on an emotion lexicon. They construct a Chinese emotion lexicon from 3 different resources:

1) Use the emotion lexicon from DUTIR1

2) Collect and use a few slang words

Keyword spotting technique for emotion recognition where a text document is taken as input and output is generated as an emotion class. Keyword spotting technique has consists of five steps. In the first step text data is converted into tokens, from these tokens emotion words are identified and detected. This technique takes text as input and in next step performs tokenization on the input text. Emotional words are identified in the next step; afterwards analysis of the intensity of emotion words is performed. Sentence is checked whether negation is presented in it or not then finally an emotion class will be found as the required output. Table 1 shows that how this technique is applied in real time chat systems.

Table 1 Keyword spotting method in real time chat system

\begin{tabular}{|l|l|l|l|l|}
\hline $\begin{array}{l}\text { Sr. } \\
\text { No }\end{array}$ & Task Description & $\begin{array}{l}\text { Emotion } \\
\text { Model }\end{array}$ & Features & Granularity \\
\hline 1 & $\begin{array}{l}\text { Emotion } \\
\text { detection in chat } \\
\text { system and } \\
\text { displaying } \\
\text { emotion using an }\end{array}$ & Ekman & $\begin{array}{l}\text { WordNet- } \\
\text { Affect DB. } \\
\text { wordNet1.6 } \\
\text { Emotional } \\
\text { weight, } \\
\text { OMCS } \\
\text { knowledge } \\
\text { base }\end{array}$ & Sentence \\
\hline 2 & $\begin{array}{l}\text { Laboratory } \\
\text { controlled online } \\
\text { chat experiment } \\
\text { to enact sadness } \\
\text { and happiness } \\
\text { and reporting } \\
\text { strategies that } \\
\text { people employ to } \\
\text { express emotions } \\
\text { in text }\end{array}$ & $\begin{array}{l}\text { Social } \\
\text { informat } \\
\text { ion } \\
\text { processi } \\
\text { ng } \\
\text { madel, } \\
\text { happy }\end{array}$ & $\begin{array}{l}\text { Linguistic } \\
\text { Enquiry and } \\
\text { word count } \\
\text { program, } \\
\text { LrWC }\end{array}$ & Sentence \\
Emotion \\
$\begin{array}{l}\text { detection } \\
\text { incorporating } \\
\text { personality factor } \\
\text { in chatting } \\
\text { system to } \\
\text { improve } \\
\text { accuracy results }\end{array}$ & $\begin{array}{l}\text { OCC } \\
\text { model, } \\
\text { Five- } \\
\text { Factor } \\
\text { (FFM) }\end{array}$ & $\begin{array}{l}\text { Open Mind } \\
\text { Common } \\
\text { sense } \\
\text { knowledge } \\
\text { base } \\
\text { (OMCS), } \\
\text { Concept } \\
\text { Net }\end{array}$ & Sentence \\
\hline
\end{tabular}

3) Collect a list of emoticons from the micro blog web site to enhance the lexicon.

This approach uses a Chinese segmentation tool to segment a Chinese micro blog text into words. Based on the constructed emotion lexicon, count the number of emotion words occurring in a text for each emotion type, and then the with the number of emotion words appearing in the text. The text is labeled as "none" when a text does not contain any emotion words. The above process also applied on sentences to get the sentence-level emotion label.

\subsubsection{Learning Based Approach}

Learning-based methods try to recognize emotions based on a previous trained classifier/results, which mapped with various machine learning classifiers such as support vector machines, specific statistic learning methods and decision trees, to detect which emotion category/class should the input text belongs.

This approach face difficulties like these methods may classify sentences into only two categories because of insufficient features other than emotion keywords, which are negative and positive. Dung et al. [9] make use the idea that emotions are related to human mental states which are caused by some emotional events. This means that the human mind starts with initial mental state and moves to another state upon the occurrence of a certain event. This idea is implemented using Hidden Markov Model where each sentence consists of many sub-ideas and each idea is treated an event that causes a transition to a certain state The sequence of events in the sentence is followed by the emotion label of the text is determined as the emotion type 


\section{International Journal of Science and Research (IJSR) \\ ISSN (Online): 2319-7064}

Index Copernicus Value (2013): 6.14 | Impact Factor (2015): 6.391

system and determines the emotion of the text. The system achieved F-score of $35 \%$ when tested on the ISEAR (International Survey on Emotion Antecedents and Reactions) dataset [10], where the best precision achieved was $47 \%$.

\subsubsection{SVM-Based Approach [8]}

SVM based approach is used as the learning model in learning-based approaches. It uses the LIBSVM toolkit 4 for multiclass emotion classification. The following three kinds of text-based features are used at document-level and sentence-level emotion classification, 1) Word Features: All the Chinese words appearing in a micro blog text or sentence are used as features. 2) Punctuation Features: Some punctuation sequences can reflect special kinds of emotions, and collect a list of such punctuation sequences as features. 3) Emotion Lexicon Features: Take the number of words of each emotion type occurring in a text or sentence as feature.

\subsubsection{Hybrid Based Approach}

This approach is based on a combination of the keyword based method and learning based method. The main advantages of this approach is that

1) It can give up higher accuracy results from training and adding knowledge-rich linguistic information from dictionaries and thesauri.

2) It will balance the high cost involved for information retrieval tasks and minimize difficulties

Yang et al. [11] presented a hybrid model for emotion classification that includes lexicon-keyword spotting, CRF based emotion cue identification, and machinelearningbased emotion classification using SVM, Naïve Bayesian and Max Entropy. The results which are generated from the above methods are integrated using a vote-based system. The system is on a dataset of suicide notes where it gains an Fscore of $61 \%$ with precision $58 \%$ and recall $64 \%$. This technique achieved relatively good results.

This section outlined the major approaches for text based emotion detection and shown how syntactic and semantic information can be beneficial for emotion detection. Current methods are lacking in in-depth semantic/analysis for detecting hidden phrase patterns and more investigations need to be done to identify, build and incorporate knowledge rich linguistic resources that have a focus on detecting emotions.

\section{Limitations}

There are few limitations in the above methods [12]

\subsection{Ambiguity in Keyword Definitions}

Using emotion keywords is a straightforward way to detect associated emotions, the meanings of keywords could be multiple and vague, as most words could change their meanings according to different usages and contexts. Even the minimum set of emotion labels could have different emotions in some extreme cases such as ironic or cynical sentences.

\subsection{Incapability of Recognizing Sentences without} Emotional Keywords Keyword-based

Approach is totally based on the set of emotion keywords. Therefore, sentences without any emotional keyword would imply that they do not contain any emotion at all, which is obviously wrong.

For example,

"I passed the GATE exam today" and "Hooray! I passed the GATE exam today"

Should denote the same emotion (joy), but the former without "hooray" could remain undetected if "hooray" is the only keyword to detect this emotion.

\subsection{Lack of Linguistic Information}

Syntax structures and semantics even have influences on expressed emotions.

For example,

"I laughed at him" and "He laughed at me"

Would signifies different emotions from the first person "es perspective. Ignoring linguistic information also poses a problem to keyword-based methods.

\subsection{Difficulties in Determining Emotion Indicators [13]}

Learning-based methods can automatically determine the probabilities between features and emotions but the methods still need keywords, but in the form of features. The most intuitive features could also be emoticons which can be seen as author"s emotion annotations within the texts. The cascading problems would be the similar as those in keyword-based approaches.

\section{Text Normalization Techniques For Resolving Short Messaging Language}

\subsection{Dictionary Substitution Approach}

Normalization of short messaging language words can be considered as a general area where our matter belongs to. There are different sub areas like sense disambiguation, text to speech synthesis and spell correction under the concern of normalizing short messaging language words. This is the traditional approach for short messaging language mapping. There were some popular web sites such as translate [14] which facilitates a service to translate short messaging language to plain text and vice versa.

\subsection{Spell Checker Approach}

This spell checker approach focuses on resolving short messaging language words to plaint text words not only by looking at just the characters, but considering contexts of that words also [18]. This approach uses edit distance to find out words confusions. For checking how suitable a particular word is for replacing, it uses context based spell checker approach. Minimum edit distance is a method used in identifying the difference between two words. For 


\section{International Journal of Science and Research (IJSR) \\ ISSN (Online): 2319-7064}

Index Copernicus Value (2013): 6.14 | Impact Factor (2015): 6.391

transforming one string to another string minimum number of edit operations are required.

Basically Deletion, Insertion and Substitution are the edit operations. While determining the value this approach uses marking schema for each edit operation. For an example, the cost for both insertion and deletion edit operations is 1 and the cost for substitution is 2. Similarly determine a value for converting each word to another word. This value is important in spell correction applications. In spell checking application the minimum edit distance of short messaging language word is calculated and using those values predict the exact word of short messaging language word as the word with minimum edit distance value [18].

In this approach use the minimum edit distance to determine wrongly interpreted short messaging language words. Text contains plain text words and short messaging language words. Consider an example the word ,tomorrowe, the most frequently used short messaging language word of it is „tmrwee, but there are many versions of word „tmrwe such as „tmrrwee, „tmowe. Thus, for these a words, predict the most possible correct word of it using minimum edit distance.

\section{Conclusion}

In this paper, the survey on existing emotion recognition approaches is done and observed that existing system make use of plain text only. This paper describes the different text based emotion recognition methods and their limitations. The problems are faced by the emotion recognition system while processing raw text which contain both plain text and short messaging language. This paper addresses the existing different approaches for resolving processing of raw textual data which contain combination of both plain text and short messaging language. These types of system are applicable for different e-contents like chat, blogs, e-learning systems etc.

\section{References}

[1] Pilar Rodriguez, Alvaro Ortigosa, Rosa M. Carro, "Extracting Emotions from Texts in E-learning Environments," Sixth International Conference on Complex, Intelligent, and Software Intensive Systems 2012.

[2] SivaramanSriram, XiaobuYuanAn, "Enhanced Approach for Classifying Emotions using Customized Decision Tree Algorithm," IEEE, 2012.

[3] Sidney K. D"Mello and Art Graesser. "Language and Discourse Are Powerful Signals of Student Emotions during Tutoring," IEEE Transactions On Learning Technologies, VOL. 5, NO. 4, 2012.

[4] Carlo Strapparava, Rada Mihalcea, "Learning to Identify Emotions in Text," SAC 08 proceedings of the 2008 ACM symposium on applied computing pages 1556- 1560.

[5] Haji Binali, Chen Wu,VidyasagarPotdar, “ Computational Approaches for Emotion Detection in Text," IEEE DEST 2010.

[6] Chun-Chieh Liu, Ting-Hao Yang, Chang-Tai Hsieh, Von-Wun Soo, "Towards Text-based Emotion Detection: A Survey and Possible Improvements," in
International Conference on Information Management and Engineering,2009.

[7] Frinken, V.; Fischer, A.; Manmatha, R.; Bunke, H. "A Novel Word Spotting Method Based on Recurrent Neural Networks" Pattern Analysis and Machine Intelligence, IEEE Transactions on Year: 2012, Volume: 34, Issue: 2 Pages: 211 - 224

[8] Wen, Shiyang, and Xiaojun Wan. "Emotion Classification in Microblog Texts Using Class Sequential Rules." Twenty-Eighth AAAI Conference on Artificial Intelligence. 2014.

[9] Dung T. Ho, and Tru H. Cao. "A high-order hidden Markov model for emotion detection from textual data."Knowledge Management and Acquisition for Intelligent Systems.Springer Berlin Heidelberg 2012.94-105.

[10] Klaus R. Scherer, and Harald G. Wallbott. "Evidence for universality and cultural variation of differential emotion response patterning." . 